04.1;04.4

\title{
Новый анализатор ионов по энергии, массе и заряду как комбинация линейного фильтра Вина и энергоанализатора с задерживающим потенциалом
}

\author{
(C) Н.А. Строкин ${ }^{1}$, В.М. Бардаков ${ }^{1,2}$, Нгуен Тхе Тханг ${ }^{1}$, А.В. Казанцев ${ }^{1}$ \\ ${ }^{1}$ Иркутский национальный исследовательский технический университет, Иркутск, Россия \\ ${ }^{2}$ Иркутский государственный университет путей сообщения, Иркутск, Россия \\ E-mail: strokin85@inbox.ru
}

Поступило в Редакцию 30 декабря 2019г.

В окончательной редакции 30 декабря 2019 г.

Принято к публикации 20 февраля 2020 r.

Комплекс последовательно расположенных линейного фильтра скоростей Вина (WLF) и энергоанализатора с задерживающим потенциалом (RFA) позволил измерять спектры ионов многокомпонентного плазменного потока по энергии, массе и заряду. Приведено описание идей и результатов испытания двух вариантов анализатора: WLF-RFA и RFA-WLF.

Ключевые слова: анализ ионов по энергии, массе и заряду.

DOI: 10.21883/PJTF.2020.10.49422.18170

Для анализа потока ионов по нескольким параметрам, например массе и энергии, используются последовательно расположенные энерго- и масс-анализаторы. Способ анализа при этом носит название тандемной спектрометрии $[1,2]$. Анализ по массе в таких приборах проводится при работе только с моноэнергетическими группами ионов исходного пучка. Другой подход к анализу ионов по энергии и массе реализуется в совмещенных энергомасс-анализаторах [3]. Прямой анализ по заряду в [1-3] не осуществляется.

Мы ставили перед собой задачу найти идею и разработать анализатор, который обеспечивал бы анализ потоков ионов по энергии, массе, заряду и при анализе по массе и заряду допускал бы возможность работы с немоноэнергетическими пучками ионов, имеющими значительный начальный угловой разброс скоростей. Создание такого анализатора инициировано необходимостью измерения пространственного распределения продуктов плазмооптического разделения многокомпонентной смеси на элементы или группы элементов. Поставленная задача, как оказалось, успешно решается при проведении анализа в тандеме последовательно расположенных энергоанализатора с задерживающим потенциалом (RFA) [4-6] и линейного фильтра Вина (WLF) [7,8]; последовательность расположения анализаторов в тандеме можно изменять на обратную: WLF-RFA.

Схема анализатора по варианту RFA-WLF приведена на рис. 1, $a$. Входная сетка $G 1$ RFA имеет нулевой потенциал и разделяет электрические поля RFA и плазмы; сетка $G 2$ убирает электроны из потока плазмы. В области $G 2-G 3$ движется только поток ионов. Фильтр Вина выполняется „коротким“: детектор размещен не в фокусе WLF, а непосредственно на его выходе. Это объясняется необходимостью иметь большую апертуру прибора. Такое свойство анализатора обязательно при регистрации ионов многокомпонентного потока, имеющих трехмерные траектории движения. WLF настроен на дрейфовую скорость $v_{0}=E / B$, которая выбирается по наиболее вероятной энергии $W_{m}$ иона, масса которого $m_{1}$ является известной или прогнозируемой. При этом ионы других масс, также движущиеся со скоростью $v_{0}$, есть в исходном спектре, ограниченном максимальной $\left(W_{\max }\right)$ и минимальной $\left(W_{\min }\right)$ энергиями.

Когда на анализирующей сетке $G 3$ RFA потенциал равен нулю, все ионы, имеющие скорость $v_{0}$, проходят через WLF на детектор. Ион с массой $m_{1}$, прошедший через WLF, имеет энергию $W_{1}=m_{1} v_{0}^{2} / 2$. На детектор пройдут и другие ионы, для которых выполняется условие прохождения $W_{k} / m_{k}=v_{0}^{2} / 2=E^{2} / 2 B^{2}$. Система регистрации будет выдавать суммарный максимальный токовый сигнал, определяемый вкладами ионов $m_{1} \ldots m_{k}$. Пусть известно, что в потоке ионов есть ионы четырех масс: $m_{1}, m_{2}, m_{3}$ и $m_{4}$, причем $m_{1}<m_{2}<m_{3}<m_{4}$. На детекторе ионы массы $m_{1}$ не исчезнут, пока тормозящим полем сетки $G 3$ RFA не будут отражены ионы массы $m_{1}$ с энергиями от исходной минимальной $W_{\min } 1$ до энергии настройки фильтра Вина $W_{1}+\Delta W$, где $\Delta W-$ абсолютное разрешение WLF по энергиям. На кривой задержки (рис. 2) $I=f\left(U_{a n}\right)$ ток в данных условиях будет определяться как $I=I_{1}+I_{2}+I_{3}+I_{4}$. При дальнейшем увеличении $U_{a n}$ ионы массы $m_{1}$ выходят из области настройки WLF, сигнал от этих ионов исчезает. На кривой задержки амплитуда тока уменьшается до уровня $I=I_{2}+I_{3}+I_{4}$. При еще бо́льших величинах $U_{a n}$ исчезнет вклад от ионов с массой $m_{2}$ и ток ионов будет определяться током частиц с массами $m_{3}$ и $m_{4}$. Ток с детектора станет нулевым при $e U_{a n} \geqslant W_{4}+\Delta W$. На кривой задержки таким образом будут видны ступеньки тока, число которых будет равно числу разных масс у ионов в налетающем потоке (четыре в нашем случае). 

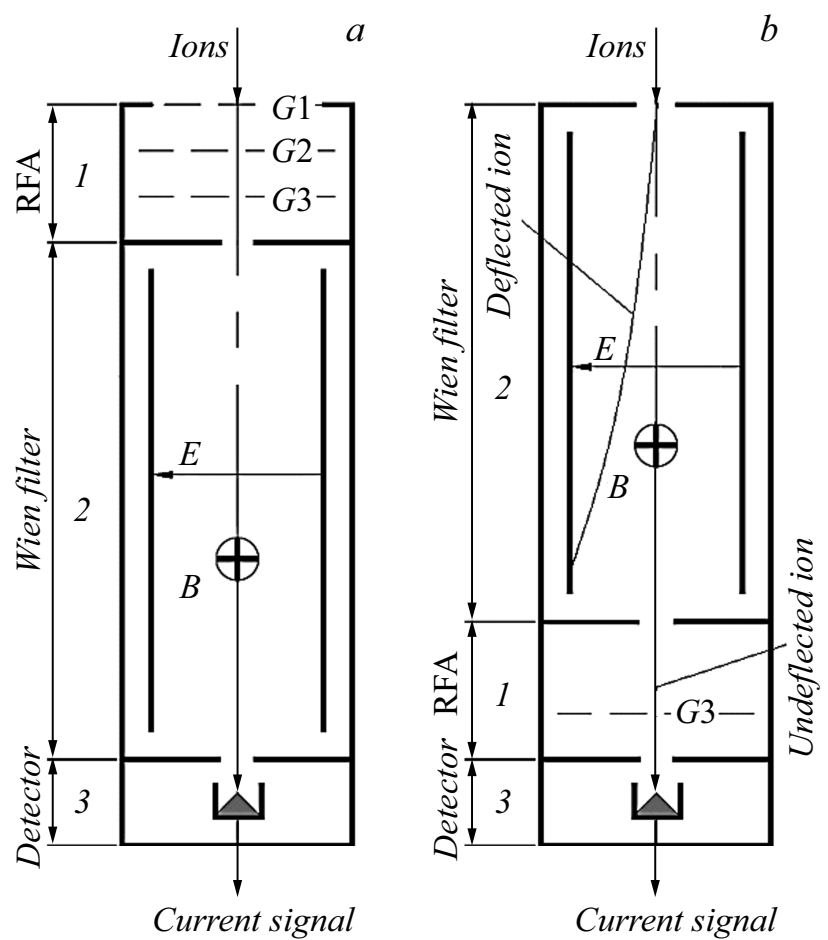

Рис. 1. Схема анализатора. $a-$ вариант RFA-WLF; $b-$ вариант WLF-RFA.

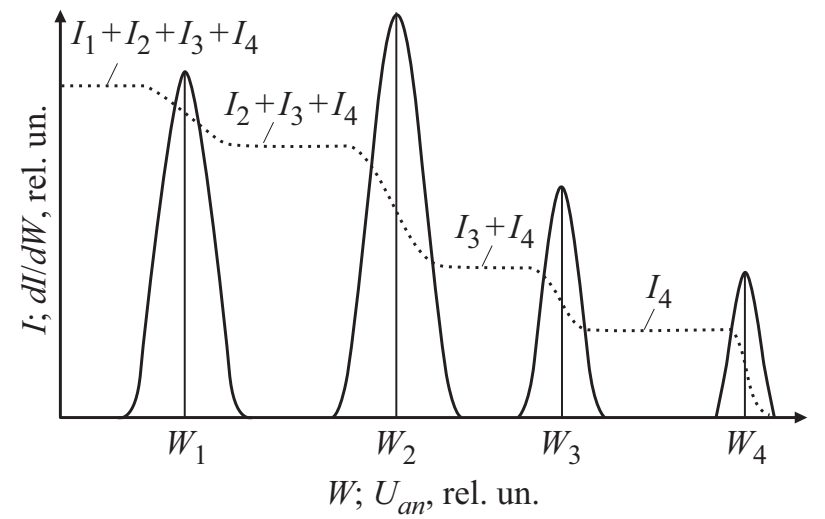

Рис. 2. Кривая задержки (пунктир) и спектры ионов четырехкомпонентного потока ионов.

Дифференцирование кривой задержки дает распределение ионов по энергиям $d I / d W$, на котором видны четыре пика ионов (начиная с самых легких) при различных энергиях, соответствующих ионам четырех масс (рис. 2). Поскольку условие прохождения ионов на детектор и величины $E$ и $B$ известны, по полученным $W_{k}$ программа обработки данных вычисляет массы ионов: $m_{k}=2 W_{k} B^{2} / E^{2}$.

При работе анализатора в варианте WLF-RFA (рис. $1, b$ ) на вход RFA поступают только ионы, поэтому сетки $G 1$ и $G 2$ здесь не нужны. Анализ по массам происходит аналогично варианту RFA-WLF.
При регистрации WLF-RFA потока ионов, имеющих широкий спектр по энергиям и разные заряды $q$ (пусть $q_{\max }=3$ в единицах заряда электрона), когда нет задерживающего потенциала $U_{a n}$ на сетке $G 3$ RFA, a WLF настроен на энергию $W_{1}$ однократно заряженного иона, на детектор проходят ионы со всеми кратностями заряда, энергии которых удовлетворяют условию прохождения; ток детектора при этом максимальный. При увеличении $U_{a n}$ ион с большим зарядом в электрическом поле RFA будет тормозиться сильнее, так как сила, действующая на ион, $F=q E_{a n}$ прямо пропорциональна его заряду $q$ и напряженности электрического поля $E_{a n}$, определяемой потенциалом сетки $G 3$. При $e U_{a n} \approx W_{1} / 3+\Delta W$ из апертуры WLF уйдут ионы с $q=3$; когда $e U_{a n} \approx W_{1} / 2+\Delta W$, уйдут ионы с зарядом $q=2$; если будет достигнуто значение $e U_{a n} \approx W_{1}+\Delta W$, то и ионы с $q=1$ выйдут из апертуры WLF. Кривая задержки RFA при росте $U_{a n}$ будет дополнена ступенями, определяемыми изменением зарядового состава ионов потока с кратностью $q>1$. При этом ионы с наибольшей кратностью заряда в результате располагаются в области наименьших энергий на спектре. Максимальная кратность заряда будет равна числу ступеней на кривой задержки. Амплитуда ступенек тока будет пропорциональна содержанию в потоке ионов с соответствующей кратностью заряда. Идентификация ионов по кратности заряда происходит в процессе программной обработки данных масс-анализа при последовательной настройке программы на ион конкретной массы.

Испытание анализатора проводилось на трехкомпонентном (неон, аргон, криптон) потоке ионов из
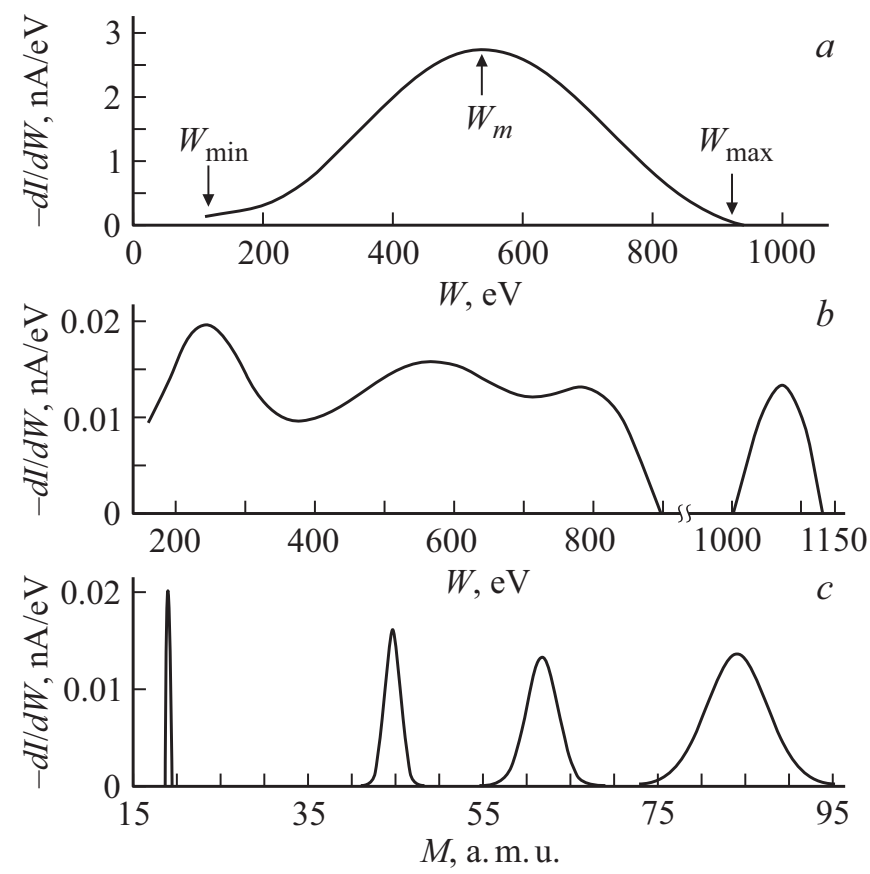

Рис. 3. Суммарное распределение ионов по энергии (a), спектр ионов по энергии на выходе тандема $(b)$ и спектр масс (c). 
Результаты анализа ионного потока по массе и заряду

\begin{tabular}{c|c|c|c|c|c}
\hline Номер пика & $W, \mathrm{eV}$ & $M$, a.m.u. & $q, e$ & Ион & Ион/W \\
\hline 1 & 241 & 18.9 & 1 & $\mathrm{Ne}^{+}$ & $\mathrm{Ne}^{+} / 258$ \\
& & 44.5 & 2 & $\mathrm{Ar}^{2+}$ & \\
2 & 569 & 44.5 & 1 & $\mathrm{Ar}^{+}$ & $\mathrm{Ar}^{+} / 510$ \\
& & 84.0 & 2 & $\mathrm{Kr}^{2+}$ & \\
3 & 787 & 61.6 & 1 & $\mathrm{Fe}^{+}$ & $\mathrm{Fe}^{+} / 714$ \\
4 & 1073 & 84.0 & 1 & $\mathrm{Kr}^{+}$ & $\mathrm{Kr}^{+} / 1071$
\end{tabular}

плазменного ускорителя TAL. WLF имел следующие характерные размеры: расстояние между пластинами конденсатора $6 \mathrm{~mm}$, расстояние между полюсными наконечниками магнитов $16 \mathrm{~mm}$, длина постоянных NeFeB-магнитов $50 \mathrm{~mm}$, ширины входной и выходной щелей $1 \mathrm{~mm}$. Средняя по длине WLF величина $B \approx 0.26 \mathrm{~T}$. Настройка WLF осуществлялась изменением величины напряженности электрического поля $E$. Диаметр входного окна аксиально-симметричного RFA равен $10 \mathrm{~mm}$ при его полной длине до детектора $15 \mathrm{~mm}$. В качестве детектора использован вторичноэмиссионный умножитель. Результаты тестирования приведены на рис. 3 и в таблице. Суммарное распределение ионов по энергии (рис. 3, $a$ ) измерено независимым RFA, размещенным на том же расстоянии от TAL, что и тандем. Параметры TAL были следующими: разрядное напряжение $900 \mathrm{~V}$, поле на аноде $0.072 \mathrm{~T}$, суммарное рабочее давление $1.4 \cdot 10^{-5}$ Torr. Набор ионов, выделенных в процессе программного анализа спектра (рис. 3,b), полученного при дифференцировании кривой задержки RFA, при напряжении настройки WLF $U_{\mathrm{WLF}}=80 \mathrm{~V}$, приведен в таблице. В последнем столбце таблицы приведены расчетные значения энергий ионов $W_{C}(\mathrm{~B} \mathrm{eV})$ при заданной настройке WLF. Кроме ионов плазмообразующих газов регистрируются ионы железа, которые появляются при ионизации атомов материала катода TAL, распыленного ионами рабочих газов. Ионы неона $(W=241 \mathrm{eV})$, аргона $(569 \mathrm{eV})$ и криптона $(1073 \mathrm{eV})$, как показал дальнейший анализ, являются однократно заряженными.

Особенностью набора рабочих газов в данном случае является примерная кратность масс: $M_{\mathrm{Kr}} / M_{\mathrm{Ar}} / M_{\mathrm{Ne}} \approx 4 / 2 / 1$, поэтому на энергиях 241 и $569 \mathrm{eV}$ программой были, „предложены“ и двукратно заряженные ионы аргона и криптона соответственно, которые при дальнейшем анализе „отбраковываются“.

Подводя итог, можно сказать, что новый анализатор расширяет функциональные возможности приборов корпускулярной диагностики плазмы, обеспечивая в одном цикле измерений получение информации как о массовом, так и о зарядовом содержании многокомпонентного немоноэнергетического потока ионов, имеющих и большой начальный угловой разброс.

\section{Финансирование работы}

Работа поддержана Минобрнауки РФ (грант № 3.6034.2017/6.7) и Российским научным фондом (грант № 18-79-00037).

\section{Конфликт интересов}

Авторы заявляют, что у них нет конфликта интересов.

\section{Список литературы}

[1] de Hoffmann E., Stroobant $V$. Mass spectrometry. Principles and applications. 3rd ed. Chichester: John Wiley \& Sons, 2007. $502 \mathrm{p}$.

[2] Münzenberg G. // Int. J. Mass Spectrom. 2013. V. 349-350. P. 9-18. https://doi.org/10.1016/j.jims.2013.03.009

[3] Астраханщев Н.В., Бардаков В.М., Во Ньы Зан, Кичигин Г.Н., Лебедев Н.В., Строкин Н.А. // ЖТФ. 2011. Т. 81. B. 9. C. $105-112$.

[4] Wei P.S.P., Kuppermann A. // Rev. Sci. Instrum. 1969. V. 40. N 6. P. 783-785. https://doi.org/10.1063/1.1684068

[5] Cui Y., Zou Y., Valfells A., Reiser M., Walter M., Haber I., Kishek R.A., Bernal S., O’Shea P.G. // Rev. Sci. Instrum. 2004. V. 75. N 8. P. 2736-2745. https://doi.org/10.1063/1.1777384

[6] Bardakov V.M., Ivanov S.D., Kazantsev A.V., Strokin N.A. // Rev. Sci. Instrum. 2015. V. 86. N 5. P. 053501. https://doi.org/10.1063/1.4920998

[7] Blase R.C., Miller G., Westlake J., Brockwell T., Ostrom N., Ostrom H., Hunter J.W. // Rev. Sci. Instrum. 2015. V. 86. N 10. P. 105105. https://doi.org/10.1063/1.4932184

[8] Wien K. // Braz. J. Phys. 1999. V. 29. N 3. P. 401-414. DOI: $10.1590 / \mathrm{S} 0103-97331999000300002$ 